\title{
Omni-Chassis Robotics: A Paradigm Shift in Thinking
}

\section{William Lovell ${ }^{\star}$}

CEO, c-Link Systems, Inc, Norway, USA

\section{Introduction}

The term Omni-robotics is very seldom used as part of our terminology speech. We have a tendency to classify our robots by manufacturer and number of axes or more appropriately degrees of freedom, or DoF. Some of us still define a robot by the early concept. A robot is a machine that handles the three D's; Dull, Dirty and Dangerous.

The word omni is defined as; all. This could lead to the calling of all 6-axis arms, omni-arms. What this means is all the arms have the same type of motion and have the same basic function; to move the end-effector from point A to B. All 6-axis robots from Kuka, ABB or Yaskawa Motoman have the same basic function. What makes them unique is the end-effector or payload. I am sure colleagues from these companies will want to talk to me about the generalization. We do not consider having a whole factory with the same robot model; we then differentiate its job by the end-effector and the software to control it. This paper will look more at the basic hardware than the complex software used to create a full functioning robot.

Since the omni-robotics term is not really used in the robotic arm industry I began using it for a new type of robot. Introducing the Omni-chassis, this is a powered robotic chassis that can carry different payloads or functioning modules. The Omni-chassis covered in this paper comes as a 6-wheel or track version. The way we usually explain this concept is like buying a garden tractor and adding the attachments you need for your yard/project. The attachments are normally called payloads because they mount onto the chassis.

\section{Omni-Chassis Sections}

This paper is broken into sections explaining the various sections of the chassis. Figure 1 is basic diagram for a 6-wheel omni-chassis.

\section{Power plant}

The power to be supplied will be dependent on chassis size, speed requirement and types of power needed. For a moderate sized chassis battery power is viable. If you need large amounts of hydraulic power, owing to the chassis size or the types of payloads to be carried, diesel or propane engine is the consideration. Choosing your powerplant one must remember to consider that the powerplant must be able to be controlled via electronics either remotely or via AI.

\section{Drive system}

The drive system depending on physical robot size can become complex. We chose to use electric drive on either wheel or track.

Some disagree on electric drive on tracks but we cannot be far off since Caterpillar Corp. is using it in some of their large bulldozers. We found that speed and torque control was easier then engine/ transmission throttling. The 6-wheel drive system has a motor per wheel in lieu of a single motor and gear/shaft or chain drive. The track drive electric motor becomes potentially large to generate the required torque, there is on motor per track. A small hydraulic system that contains an electrically driven pump is also viable for the track system on medium and small chasses.
Basically, the designer of the robot system has to either take all into account or ensure you communicate what is needed to the team.

\section{Chassis design}

Designing a chassis is one of the few times that some mechanical or systems engineer gets a chance to show off their ability. Here the specifications that need to laid out are the final size, capability, the chassis needs and size, mounting or style of payload packages.

With these known the engineers can move forward with the shape. Nowadays a lot of small vehicles are becoming esthetic in design with nice rounded corners and edges. The materials to be used are dependent again on the specified function requirement. Composites and aluminum are becoming more prevalent in usage because of the reduced weight. High end steel alloys are still used in a lot of cases. Some material choice is based on the company's production ability. If you are not prepared to undertake the adding of new equipment and training then you stay with what can be worked with. The only other option is to subcontract the work, which in itself opens a new batch of issues [1].

\section{Electronics}

Of all the design work the electronics is the most complex. Yes you can use your COTS if it will suit your needs, utilizing a PLC (Programmable Logic Controller) is not really recommended due to the inherent latency of the units scan time. First off, the type of robot chassis needs to be decided upon; remote control wire, remote control radio/Wi-Fi, remote control with built in job program or our favorite, autonomous. The autonomous type of electronics will be used in this paper because the other configuration types are inherently built-in creating a complex system.

The control systems engineer needs basic specs on everything that needs to be controlled and/or monitored. The system has been broken down into the following groups:

1. Main processor

2. Guidance/navigation

3. Motion

4. Power management

5. Safety

${ }^{*}$ Corresponding author: William Lovell, CEO, c-Link Systems, Inc, 212 Eddie Kahkonen Rd. Norway, USA, Tel: 207-515-0323; E-mail: wlovell@c-linksystems.com

Received September 29, 2017; Accepted October 11, 2017; Published October 16, 2017

Citation: Lovell W (2017) Omni-Chassis Robotics: A Paradigm Shift in Thinking Adv Robot Autom 6: 175. doi: 10.4172/2168-9695.1000175

Copyright: (c) 2017 Lovell W. This is an open-access article distributed under the terms of the Creative Commons Attribution License, which permits unrestricted use, distribution, and reproduction in any medium, provided the original author and source are credited. 


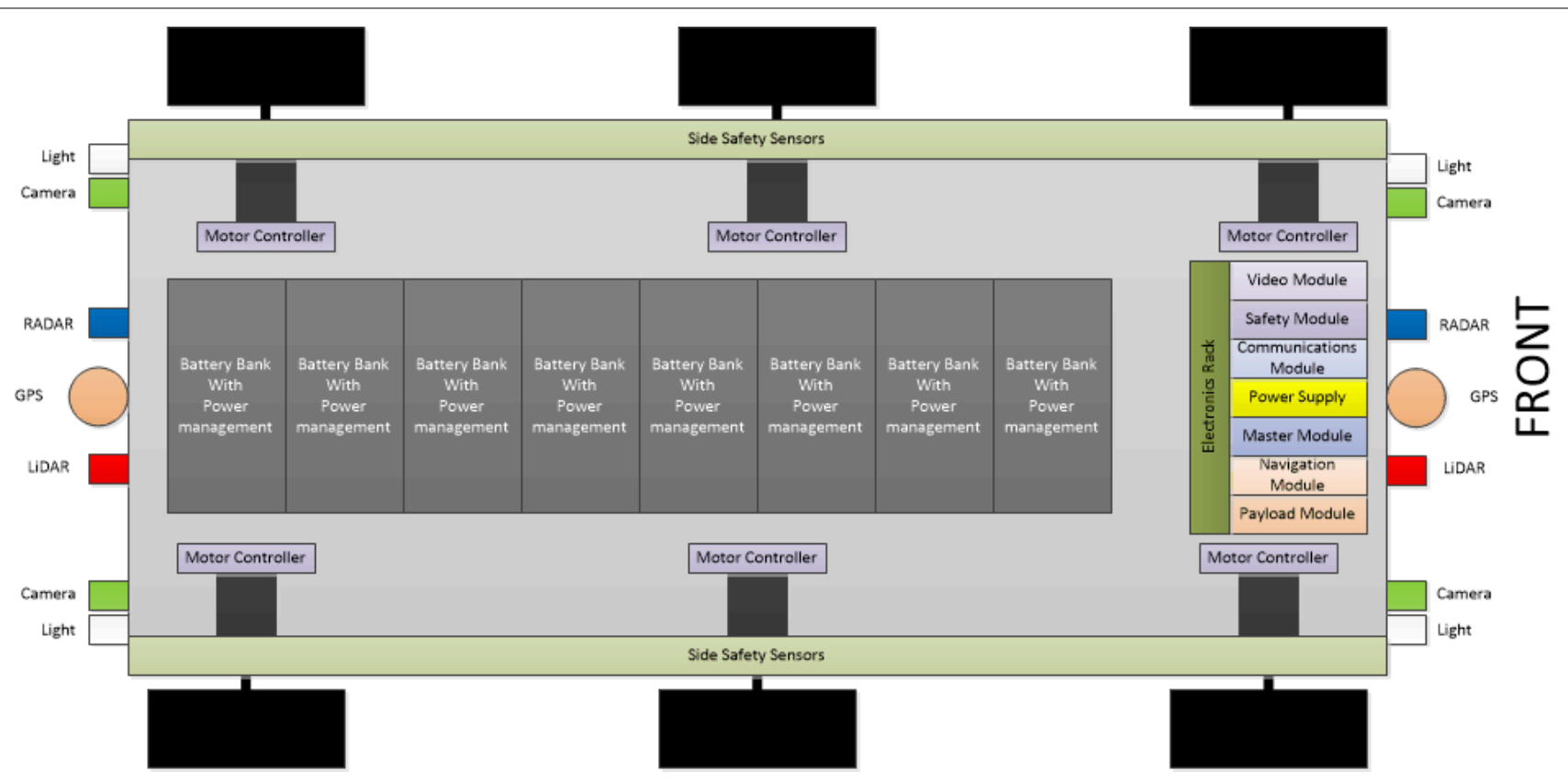

Figure 1: Basic diagram for a 6-wheel omni-chassis.

6. Communications

7. Payload control

\section{Main processor}

This would be the master or only processor. Again, depending on the overall needs this could be a large-scale CPU or a MCU. In some cases, a distributive processor system will better fit overall control. For those that do not understand, a distributive system has processors that handle specific jobs, i.e., motor control, guidance/navigation, power management and so forth. All of the sub-processors report to the master.

\section{Guidance/Navigation}

Guidance: The directing of the motion or position of something. The term is usually used in reference to a missile.

Navigation - the process or activity of accurately ascertaining one's position and planning and following a route.

For autonomous vehicles a combination of both is usually used. The navigation process can have many components. The following combination is currently in use:

1. Dual GPS receivers

2. Wheel/track RPM/position indicators

3. LiDAR

4. RADAR

5. Ultrasonic range sensors

6. 3-axis gyro

7. 3-axis accelerometer

8. Cameras.

The data from all these components is combined to give, in this case, a position accuracy of $\pm 1 \mathrm{~cm}$. The guidance section takes the position information and compares it to the last navigation set and adjusts the drive motors accordingly. Basic navigation is a math intensive action, hence the need for a dedicated processor. The data field or point cloud generated by either the LiDAR or RADAR requires a dedicated processor also. This whole guidance/navigation system requires a minimum of 2 and preferably 3 processors. Since this is a complex undertaking for the purpose of this paper it will stop here.

\section{Motion}

This is basically control of the drive motors. I have found that an MCU per motor to be most effective. This allows control and monitoring of a motor up close and a reduced MCU cost. There are MCUs designed for the specific purpose of motor control. Mounting the controller on or next to the motor reduces line loss or electrical noise interference. Some of the monitored are; motor RPM, motor current, motor temperature and motor voltage. Thus, the master processor or guidance processor communicates the chassis speed to the motor MCUs, which allows each motor to self-adjust actual motor drive. Information gathered maintains the motor's health and the overall performance of the chassis. Examples; MCU sees a large drop in motor current with constant RPM - wheel is slipping, track drive sprocket has constant RPM with a drop in motor current - track break.

\section{Power management}

Primarily used on the battery system with a minor application for fuel powered engines. For battery systems you are looking at the current draw per battery bank and by utilizing relays can isolate a bank that appears to be drained or having issues. It would also handle the recharge of each bank of batteries. It will also notify the master of length of time left so the master can notify a human for service. For fuel engines it would maintain constant output RPM and monitor fuel consumption, once again notifying for service when needed.

\section{Safety}

Safety is forgotten in a lot of cases. The protection is for the robot 
from humans and other dangers. For an autonomous chassis I found that a ring of short range ultrasonic sensors worked well. They notified the master processor of the approach of humans so payload and chassis motion could be suspended. Humans are a danger. Another set of sensors are the end-of-world units, these are looking down at an angle so as to prevent the chassis from falling into a hole or going off a cliff. Also included are flashing lights, data extraction from the cameras and kill switches (both physical on the robot and via RF).

\section{Communications}

Basically communications is the ability to move data between two points. While a full autonomous robot does not need human interaction monitoring the system for issues is still important. Communications is accomplished via one of the following methods: copper cable (tethered), fiber-optic cable, RF or Wi-Fi. Wi-Fi is the preferred method of educational institutes due to high availability and low cost. Field industry leans more towards RF having a better range and security. The communications link can be used to monitor the health and status of the chassis/payload, see what the cameras are seeing and even command change in action. With added security the software could be updated on-the-fly [2].

\section{Payload control}

The payload control can be implemented in many fashions. For some putting the controls on the payload is the easiest. This would require a single data cable to communicate with the master processor, and a power cable.

I developed a different approach to this. I use a re-configurable FPGA that knows what payload is attached and configures itself accordingly. Because the FPGA has a built-in processor it also knows what software to load for all the controls. This approach saves rebuild or redesign of circuit cards that are mounted on the payload. There is also less chance of control electronics being damaged while the payload is functioning.

\section{Usage examples: Chassis with Payload}

All of the examples are designed to fit the same omni-chassis.

\section{Dump body}

Figure 2 is a rear image of a dump unit. The most basic payload is the dump body. The chassis has a dump body allowing it to move material from one point to another. This configuration is ideal for construction sites or large landscaping projects. For open pit mining a large version would be needed, this type has already been developed by
Volvo CE, the units are named HX01 and HX02.

\section{Clean up}

Figure 3 is a clean-up system for debris; the unit is a hybrid combining a dump body and a 6 -axis arm. The dump is $60 \%$ the length of the omni-chassis (could be longer but would overhang the rear of the omni-chassis. The other $40 \%$ is taken up with a 6 -axis arm utilizing different end-effectors. For debris cleanup a 5 or 6 finger end-effector would be used to move material to the dump. Once the dump was full it would trundle off to a set point to dump the load. The advantage of this payload is it can function day or night and rain or shine, it does not care [3].

\section{Disaster SaR (search and rescue)}

Figure 4 depicts an earlier version of a Disaster SaR. To date this is the most complex payload ever designed. It covers $100 \%$ of the chassis with $50 \%$ being additional batteries. The other $50 \%$ contains two 6 -axis arms working together on moving debris. The end-effectors can be change on-the-fly by the control system from a tool rack, thus allowing for holding and cutting simultaneously. Coupled with the arms are a pair of extendable boom arms, one has a color camera and the other a FliR. The FliR allows the robot to see hot spots and evaluate the potential for human life.

\section{Conclusion}

The concept of an omni-chassis, autonomous or otherwise, is to allow companies, agencies and individuals the ability to take advantage of a robot with multifunction ability at a lower cost. A single chassis can handle multiple jobs where as the alternative is multiple robots, each built for a specific job at a higher cost.

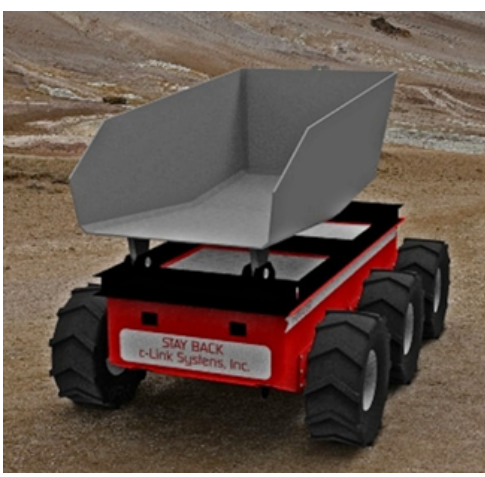

Figure 2: Dump body.

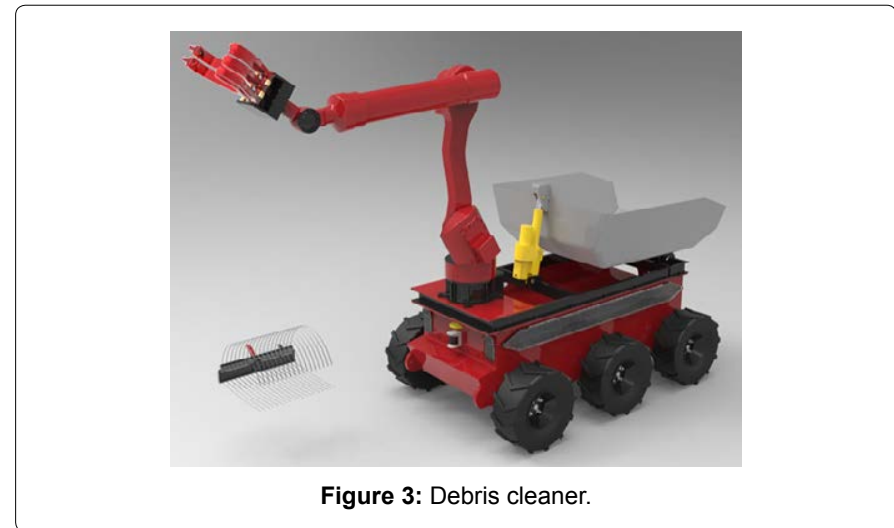

Figure 3: Debris cleaner.

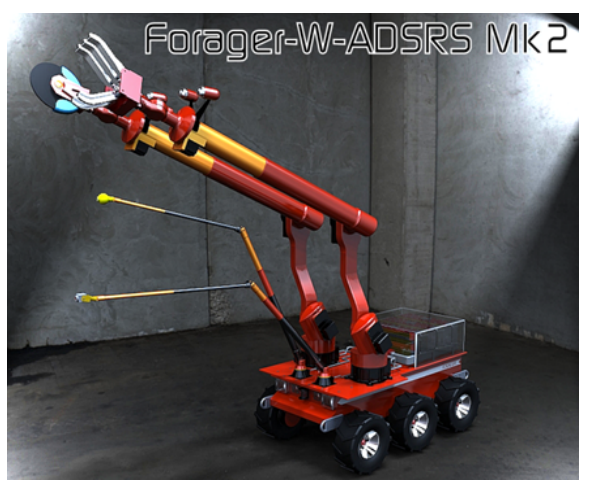

Figure 4: Disaster SaR. 


\section{References}

1. Lovell W (2007) c-Link Systems Introduces New Robotic Vehicle Targeted at Students, Hobbyists. Business Wire, A Berkshire Hathaway Company.
2. Suhas HK (2000) Quality to Virtual Corporation: An Integrated Approach. CRC Press.

3. Huang S, Chen D, Gong H (2012) Research on the Impact of the Number of Wheels and Layouts to the Performances of Omni-Directional Chassis. ICIRA 2012: Intelligent Robotics and Applications, pp: 258-267 